\title{
REVIEW
}

\section{Chemistry in nanochannel confinement}

\author{
Han J. G. E. Gardeniers
}

Received: 8 December 2008 / Revised: 29 January 2009 / Accepted: 2 February 2009 / Published online: 21 February 2009

(C) The Author(s) 2009. This article is published with open access at Springerlink.com

\begin{abstract}
This review addresses the questions of whether it makes sense to use lithographically defined nanochannels for chemistry in liquids, and what it is possible to learn from experiments on that topic. The behavior of liquids in different classes of pores (categorized according to their size) is reviewed, with a focus on chemical reactions and protein dynamics. A number of interesting phenomena are discussed for nanochannels with feature sizes that are manufacturable with modern photolithography-based fabrication technology. The use of spectroscopic methods to investigate chemistry in nanochannels, where both spectroscopic method and nanochannels are integrated into a single device, will be evaluated.
\end{abstract}

Keywords Water · Spectroscopy · Instrumentation · NMR · ESR $\cdot$ Nanoparticles $\cdot$ Nanotechnology $\cdot$ Microfluidics · Microfabrication

\section{Introduction}

In this review we investigate answers to the question of whether artificial nanochannels possess the potential for fundamental studies and applications in the field of chemistry in liquids. By "artificial" we mean that they are fabricated by "top-down" techniques involving photolithography and micromachining. The typical dimensions of the nanochannels of interest range from a few $\mathrm{nm}$ to a few hundred $\mathrm{nm}$, which is typically also

H. J. G. E. Gardeniers ( ()

Mesoscale Chemical Systems,

MESA + Institute for Nanotechnology, University of Twente,

P.O. Box 217, 7500 AE Enschede, The Netherlands

e-mail: j.g.e.gardeniers@utwente.nl the range of pore sizes available in porous materials like zeolites, membranes, polymers, and porous glasses and minerals, which are applied or studied in various fields such as filtration and sorption, petroleum engineering, soil research, heterogeneous catalysis, chromatography and size-exclusion separation techniques, as well as biochemical engineering and controlled-release medicine. In catalysis, pores are categorized according to their mean size as [1]: i) "micropores," with diameters $<2 \mathrm{~nm}$; these are close to the size of small molecules, and examples include zeolites, carbon nanotubes and aquaporins in cell membranes; ii) "mesopores," between 2 and $50 \mathrm{~nm}$, such as the pores in anodically fabricated porous silicon or the pores in particles used in chromatography; iii) "macropores," $>50 \mathrm{~nm}$ diameter. This division into pore classes makes sense, as we will see below when we discuss the phenomena observed in these pores, although the terminology seems awkward when compared to what in micro and nanotechnology are referred to as "nano," "micro" and "macro." Channels with diameters of $<1000 \mathrm{~nm}$ are usually called "nanochannels," while channels between $1 \mu \mathrm{m}$ and $1 \mathrm{~mm}$ are considered "microchannels;" more specifically, these are channels with hydraulic diameters that are much smaller than the Laplace constant (which scales surface tension to gravitational forces, and which is on the order of $1 \mathrm{~mm}$ for water at atmospheric conditions and room temperature). Everything larger than $1 \mathrm{~mm}$ is (at least among nano and microsystems aficionados) usually considered "the macroworld."

Molecules confined within pores with widths of a few molecular diameters can exhibit physical behavior that is quite different from that observed in a bulk fluid. When the pore width is on the order of the range of the intermolecular forces, a large fraction of the confined molecules will experience a reduction in the number of nearest-neighbor 
molecules, leading to large shifts in phase coexistence curves and a lowering of critical points [2]. Furthermore, the presence of wall forces in the pores, and the resulting competition between fluid-wall and fluid-fluid forces, can lead to different phase behavior from that found in the bulk fluid, like layering and shifts in phase transitions. The new physics that results from finite-size effects, varying dimensionality, and surface forces will also have implications for chemical reactions carried out in the confinement of a nanochannel, as we will discuss in this review.

Because state-of-the-art micro- and nanofabrication techniques allow the development of nanostructures with well-defined dimensions in a planar format and in large ordered arrays with a small size distribution, artificial nanochannels may serve as excellent experimental model systems for studying the properties of pores and comparing the results of such studies with those obtained from computational and theoretical modeling. State-of-the-art fabrication technology has already arrived at the small dimensions of the "micropores" mentioned above; for example, electron beam lithography achieved a resolution limit of $1 \mathrm{~nm}$ some 20 years ago [3]. Besides the dimensional definition, another advantage of the micro/ nanofabrication approach is the planar format, which is very suitable for the integration of analytical tools that can be scaled, using modern fabrication tools, to match the dimensions of the nanochannels (or rather, scaled to the typical range of the physicochemical phenomena occurring in the nanochannels). Integrated analysis provides unprecedented possibilities when studying the chemical processes occurring in nanoconfinement in situ. This in situ analysis allows the verification of theoretical pore models and thus advances our fundamental understanding of the chemistry that occurs in porous materials.

\section{Phase equilibria in nanochannels}

Confinement of matter on the nanometer scale induces phase transitions that are different from the ones observed in bulk systems [2]. This is usually attributed to fluid-wall interactions, particularly at the smallest geometries. For nanochannels or pores a few tens of nanometers in size, fluid-fluid interactions interplay with fluid-wall interactions, leading to a phenomenon called capillary condensation that is a common gas-liquid transition which is displaced towards lower pressure according to the Kelvin equation.

As an example of phase transition shifts in confinement, in work in which time-resolved optical experiments were used to study the dynamics of liquid oxygen in pores with diameters of between 2.2 and $18.7 \mathrm{~nm}$ [4], it was experimentally found that a supercooling of almost
$20 \%$ below the bulk freezing point is obtained in the smallest pores. The freezing-point depression follows the relationship:

$\Delta T=\frac{2 \Delta \sigma V_{\mathrm{m}} T_{0}}{\Delta h_{\mathrm{f}} R_{\mathrm{p}}}$

where $\Delta h_{\mathrm{f}}$ is the heat of fusion, $T_{0}$ is the bulk freezing point, $V_{\mathrm{m}}$ is the molar volume, and $\Delta \sigma$ is the difference between the frozen-solid/wall and liquid/wall interfacial energies. The relation was also found to hold for ethanol, and is a direct result of the following equation [5]:

$\Delta T=\frac{2 \alpha_{\mathrm{LS}} V_{\mathrm{S}} T_{0} \cos \theta}{\Delta h_{\mathrm{f}} R_{\mathrm{p}}}$

where $\alpha_{\mathrm{LS}}$ is the surface energy between liquid and solid, $\theta$ is the contact angle between liquid, solid and the wall of the pore, and $V_{\mathrm{s}}$ is the molar volume of the solid.

A similar relationship holds for the boiling-point elevation in pores, and the relation in that case turns out to be a combination of the Clausius-Clapeyron and Kelvin equations. The latter predicts that the boiling temperature for water in pores a few $\mathrm{nm}$ wide may rise to above $300^{\circ} \mathrm{C}$. Such high liquid temperatures, far above the bulk boiling point, may not be realistic in practice, but even an increase of a few tens of degrees in the boiling point would lead to chemical reactions with orders of magnitude faster kinetics - very similar to the effect obtained in a pressurized liquid, but without an external pressure source and all the precautions that need to be taken in that case. It should also be pointed out that channels a few $\mathrm{nm}$ in diameter are very difficult to achieve by state-of-the-art machining, as will be discussed in more detail in a following section. Exploiting the above effects in an engineered device therefore requires the use of microporous materials.

There has been some debate about the pore size at which the Kelvin equation may still be valid. It was experimentally determined that the Kelvin equation holds down to $9 \mathrm{~nm}$ for water [6], and down to ca. $4 \mathrm{~nm}$ for organic liquids [7]. In fact, subsequent largely theoretical work (see $[2,8]$ for reviews) has shown that a shift of the critical temperature of the fluid confined in pores of a few nm may arise that scales as:

$\frac{T_{\mathrm{c}}-T_{\mathrm{cp}}}{T_{\mathrm{c}}} \leq \frac{\sigma}{r_{\mathrm{p}}}$

where $\sigma$ and $r_{\mathrm{p}}$ are the Lennard-Jones size parameter and the pore radius, respectively, and $T_{\mathrm{c}}$ and $T_{\mathrm{cp}}$ are the bulk and radius critical temperatures, respectively [9]. As can be seen from Eq. 3, the shift is positive (i.e., the critical temperature is lowered by the nanoconfinement), and so the critical temperature becomes more accessible for experimentation since the high pressures that are commonly 
required for supercritical conditions are no longer needed (nanoconfinement also results in a lowering of the critical pressure [10]). Since supercritical conditions can be very attractive for chemistry [11], the use of nanochannels may provide a pathway to new chemistry. Note, however, that the critical shift will only be substantial for a very small pore radius, such as that attainable in zeolites. For example, the critical point of $\mathrm{CO}_{2}$ in $\mathrm{MCM}-41$ pores shifts from a bulk value of $304.21 \mathrm{~K}$ to a value of $195 \mathrm{~K}$ at $r_{\mathrm{p}}=1.8 \mathrm{~nm}$, and to below $161 \mathrm{~K}$ for an $r_{\mathrm{p}}$ of $1.2 \mathrm{~nm}$ [12]. These data also show that the dependence of the critical temperature on pore radius is very steep, which places severe constraints on pore size control and pore size distribution in practical applications, like the supercritical chemistry mentioned above.

One very special case, in a broad sense, is water. The importance of water to life can hardly be overemphasized, and the common belief nowadays is that life cannot exist without water [13]. Bulk water has unique properties that derive from its hydrogen-bonded network. In everyday life, though, water is often present trapped in small cavities, and this is why much effort has been devoted to describing the effects of confinement on the structure and dynamics of water.

Although the differences between the properties of bulk and confined water that emerge from different experimental methods are sometimes misleading, it is now understood that nanoconfinement of water results in suppression of the molecular rotational motions near the confining surface, within a layer of thickness $0.4-0.8 \mathrm{~nm}$ (see, e.g., [14]). Much of the latest work on water in nanoconfinement focuses on structures with diameters in the 1-2 nm range, and uses carbon nanotubes as the main theoretical and experimental model because of the similarities in terms of dimensions and hydrophobicity to pores in biological systems, like the aquaporin water channels in cells [15, 16]. The structure arising from this work is one in which water exists in a two-phase system, with a fourfoldcoordinated ice sheet wrapped into a cylinder near the nanotube wall, and a chain of water molecules along the tube axis, which retains fluidity down to $50 \mathrm{~K}[17,18]$. This chain of water, or "single file," was predicted by molecular dynamics simulations for water in carbon nanotubes, aquaporins and for pores in zeolites (see [19] for a review).

Other work in this area on confinement in pores of similar dimensions $(2.1-4.5 \mathrm{~nm})$ discusses the orientational dynamics of weakly wetting, strongly wetting and networked liquids, and concludes that in all cases the global (i.e., averaged over the nanochannel volume) dynamics of the confined liquids are slower than those in the bulk. This arises from a number of different mechanisms: geometrical reorientation constraints at the pore surface, a change in the hydrodynamic volume for the reorientation of molecules off the pore walls in weakly wetting liquids, chemical interactions with functional groups on the pore surface in strongly wetting liquids, and interference with long-range cooperativity in networked liquids [20]. It was found that molecular motions near the pore surface slow down; on the other hand, if the interactions between the molecules and the surface are weaker than the interactions between the molecules in the bulk liquid, they accelerate [21]. The unusual dynamics of confined water may be ascribed mainly to the partial destruction of hydrogen bonds, which decreases the cooperativity of molecular motions in the liquid. Similar effects have also been reported for methanol and ethanol [21], which also are hydrogen-bonded liquids. Because of the latter, the effect of confinement on the dynamics of capillary-condensed methanol molecules was found to be stronger than in acetonitrile [21, 22].

Surprisingly, it was reported that capillary-condensed water also shows suppressed molecular motions at room temperature in nanochannels tens of nanometers in size. Particularly worthy of note in this respect is the work of Tsukahara et al. [23], who used NMR (nuclear magnetic resonance) to study confinement effects on the structure and motion of water molecules in channels 295-500 $\mathrm{nm}$ in equivalent diameter. The chips containing these nanochannels were introduced into a commercial $5 \mathrm{~mm}$ NMR sample tube and measured at $300 \mathrm{MHz}$ proton resonance. NMR is particularly well suited to the study of inter- and intramolecular motions and interactions, while at the same time the method provides the possibility of identifying chemical species. It was found from the NMR data (in particular the spin-lattice relaxation values, see Fig. 1) that the water in the nanochannels retains a four-coordinated hydrogen-bonded structure, translational motions slow down, proton mobility is enhanced, the proton-charge distribution is localized along the hydrogen-bonding chains, and a chemical exchange of protons takes place between water and water adsorbed on surfaces. To assess the relative contribution of water-surface interactions, the authors also modified the nanochannel surfaces with hydrophobic Si $\left(\mathrm{CH}_{3}\right)_{3}$ groups. The spin-lattice relaxation time constants of water confined in the modified channel were found to increase at around $1000 \mathrm{~nm}$, a higher value than for the unmodified case. Although the interactions of water molecules with the $\mathrm{CH}_{3}$ groups were weaker than those with the $\mathrm{OH}$ groups, the size-confinement effect appeared stronger in the hydrophobic case than in the hydrophilic case.

\section{Transport in nanochannels}

As derived from the Hagen-Poiseuille equation, the pressure drop needed to refresh the volume of a tubular 


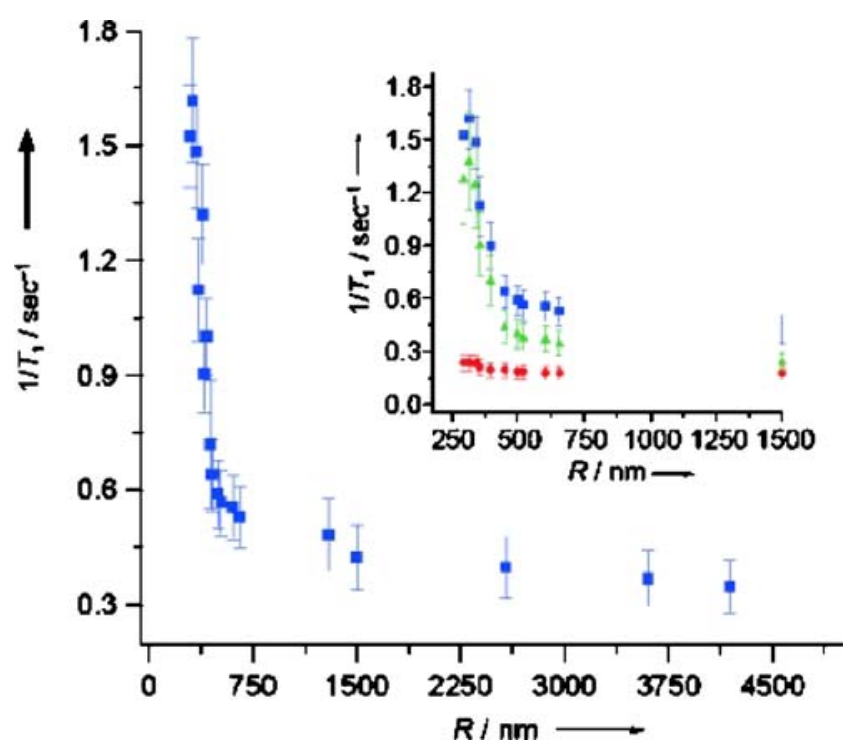

Fig. 1 Size dependence of ${ }^{1} \mathrm{H} 1 / T_{1}$ values (squares) of confined water at $300 \mathrm{MHz}$ and $22{ }^{\circ} \mathrm{C}$ ); the inset shows the size dependences of intermolecular translational (triangles) and intramolecular rotation (closed circles) motions obtained from the experimental $1 / T_{1}$ values (squares) [23]

nanochannel of radius $R$ and length $L$ once every second is given by:

$\Delta P=8 \mu\left(\frac{L}{R}\right)^{2} \mathrm{~s}^{-1}$

where $\mu$ is the dynamic viscosity. It is possible to fabricate nanochannels that have aspect ratios $L / R$ of up to $10^{5}$ (e.g., a channel of radius $10 \mathrm{~nm}$ and length $1 \mathrm{~mm}$ ). For water (with $\mu=0.001 \mathrm{~Pa} \mathrm{~s}$ ), the corresponding pressure drop in such channels would be $80 \mathrm{MPa}$ or ca. $800 \mathrm{bar}$, and the flow cwould be a. $20 \mathrm{fL} / \mathrm{min}$. Although this flow is far below the limits of available commercial pumping equipment, such a pressure is not unrealistic [24]. Putting tens or hundreds of these channels in parallel, or making them slitlike, would bring pressure-driven flow through such nanochannels within reach.
However, in the above we have assumed that flow through nanochannels can be described by the HagenPoiseuille equation. It turns out that liquid-filled channels of simple geometry, with smooth walls and with diameters $>2 \mathrm{~nm}$ can indeed be described using classical NavierStokes treatments [25]. However, in discussions of flow in nanochannels, one must always take the effects of wall charge on transport into account. Much of the work on this topic has recently been reviewed [26, 27], and we refer the reader to these papers for more detail. Summarizing their findings, since the surface-to-volume ratio is high in nanochannels, surface-charge-governed transport becomes prominent. This leads to charge selectivity and exclusion of ions from the channel (see Fig. 2), an effect that is most pronounced if the Debye screening length, or electric double layer (EDL), is comparable to the smallest dimension of the nanochannel, leading to EDL overlap (see [28] for a review of theoretical modeling of this phenomenon). Such charge-selective effects were first described in membrane filtration [29], but have recently also attracted great interest because of a similarity to transmembrane protein and potassium channels (see, e.g., [30]). Furthermore, charge exclusion has been used directly in a chromatographic application [31] where pressure-driven flow occurs through nanochannels with EDL overlap, causing the co-ions (see Fig. 2) to move faster than the counterions, resulting in chromatographic separation. When applied to DNA separations, efficiencies of more than 100,000 plates per meter were obtained with less than $1 \mathrm{pL}$ of sample consumed and less than $0.1 \mathrm{~nL}$ of waste generated.

An exciting application of the ion-exclusion effect in nanochannels is a new type of proton-exchange membrane that can be used, for example, in miniaturized fuel cells [32]. In this work an array of parallel channels of height $50 \mathrm{~nm}$ that is filled with an acidic solution acted as a super proton conductor, because the proton conductivity inside the channels increased by orders of magnitude due to EDL overlap. To increase the power output (the one-dimensional
Fig. 2 At high ionic strength the electrical double layer, EDL (shaded in gray), is thin, which allows co-ions and counterions to pass through the nanochannel. At low ionic strength the electric double layers (almost) overlap, resulting in charge selectivity. From [26]
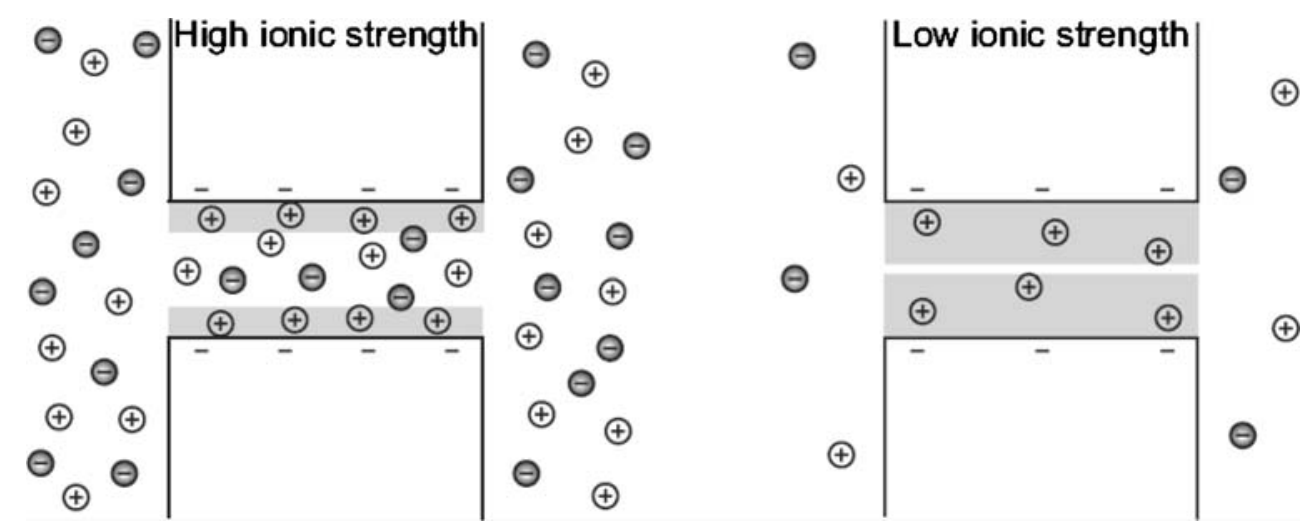
channel array presented gave an output of $3 \mathrm{nW}$ ), the number of channels should be increased; if this is done, the authors calculate that an entire fuel cell assembly with a volume of $10 \times 10 \times 10 \mathrm{~cm}^{3}$ could generate a power of more than $100 \mathrm{~mW}$.

Another interesting finding with respect to charge effects in nanochannels was reported by Schoch et al. [33] — namely that nanofluidic channels can be used to enhance surface binding reactions, since the target molecules are confined to surfaces coated with specific binding counterparts and the molecules can be steered into the nanochannels via pressuredriven or electrokinetic flow. Monitoring the nanochannel impedance allows sensitive electrical detection of low analyte concentrations within response times of $1-2 \mathrm{~h}$, which is 54 times faster than diffusion-limited binding.

This brings us to the topic of the diffusion of molecules in confined geometries, which is quite different from diffusion in the bulk. In channels a few $\mathrm{nm}$ in diameter, confinement may lead to an effect that is called "single-file diffusion," i.e., the restricted propagation of particles that cannot pass one another. The occurrence of this phenomenon is reflected by a change in the time dependence of the mean particle displacement in comparison with ordinary diffusion. This was theoretically described by Levitt [34], who showed that under single-file conditions (the studied system involved one-dimensional collisions of hard rods with a random background), the mean displacement of particles goes as time $e^{1 / 4}$, rather than the time ${ }^{1 / 2}$ behavior expected for diffusion according to Fick (i.e., the flux of material in a concentration gradient imposed on the system). Direct evidence of the expected time dependence of single-file diffusion was first provided by diffusion measurements performed with pulsed field gradient nuclear magnetic resonance (NMR) in zeolites [35].

Diffusion in zeolites has recently been reviewed by Smit and Maesen [36]. These authors argue that one should take care when comparing diffusion coefficients; for one thing, they might not have the same meaning: the transport diffusion coefficient used in Fick's law and based on the flow of material in a concentration gradient is intrinsically different from the diffusion coefficient obtained from the NMR experiments mentioned above, in which a particle is labeled and the attenuation of the label signal is determined; the latter diffusion coefficient relates to the self-diffusion coefficient, which is a measure of the average mean-square displacement of a particle in a given time interval. The basic difference between the two arises from the fact that the first (Fickian) diffusion coefficient arises from the collective behavior of many molecules due to a gradient, whereas the second is a property of an individual molecule resulting from Brownian (i.e., thermal molecular) movement. The differences between these diffusion coefficients disappear for low loading (i.e., particle density); for high loading the differences can be quite large. A more extensive description can be found in the paper by Krishna and Wesselingh [37].

The pore size in zeolites (between 0.2 and $1 \mathrm{~nm}$ ) is close to the sizes of small molecules like $\mathrm{CH}_{4}$ or $\mathrm{CO}_{2}$, and can be tuned by ion exchange in the zeolite matrix. Because of this similarity in size, it is no surprise that the diffusion coefficient of methane can vary by as much as 6-10 orders of magnitude, depending on the pore topology. A very large difference in diffusion coefficient (by a factor of $10^{8}$ compared to the coefficient in bulk water) was also observed for rhodamine $6 \mathrm{G}$ dye in $2.9 \mathrm{~nm}$ wide and $4.8 \mu \mathrm{m}$ long silica nanochannels arranged in an array [38]. The results obtained on silica nanochannels and zeolites, which are both hydrophilic materials, are quite different from the data reported for diffusion in carbon nanotubes $7 \mathrm{~nm}$ in diameter, where the diffusion of water and hydrocarbons was significantly faster than in solution [39]. This was attributed to the hydrophobicity of the carbon wall, which leads to an effectively frictionless surface inside the CNT (expressed in slip lengths of 40-70 $\mu \mathrm{m}$ ).

These molecular sized pores provide special opportunities for shape-selective chemistry, a topic discussed in the following section. One interesting term that is worth discussing briefly here is resonant diffusion. This is the effect observed in zeolites where the diffusion coefficient exhibits a periodic dependence on the number of carbon atoms in alkanes, a phenomenon that was experimentally proven in LTL and ZSM-12 zeolites [40].

Summarizing the discussion up to this point, it now has been established that in nanochannels of almost-molecular dimensions, say below $5 \mathrm{~nm}$ in diameter, effects like singlefile transport play a role. Examples include water in carbon nanotubes [18], organic molecules in zeolite pores, and potassium ions in membrane channels [41]. For nanochannels of a somewhat larger size, deviations between the diffusion coefficient in the bulk and the ones in the channels have frequently been reported. For example, work by Martin et al. [42] on diffusion through microfabricated silicon-based membranes with arrays of uniform channels with widths of $7 \mathrm{~nm}$ to $50 \mathrm{~nm}$ and of length $45 \mu \mathrm{m}$ demonstrated that non-Fickian behavior (i.e., the flux through the nanochannels does not decrease exponentially with time as predicted by Fick's law, but is instead constant for a certain period of time) occurs as the pore width approaches the hydrodynamic diameter of the solute (e.g., at a channel width of $20 \mathrm{~nm}$ for interferon and in a 13-nm-wide channel for albumin). Fickian diffusion is seen for a pore size of $26 \mathrm{~nm}$ or more. The non-Fickian behavior is attributed to constrained movement due to the walls of the nanopore.

Kaji et al. [43] performed single-particle tracking experiments in nanopillar (height: $400 \mathrm{~nm}$, spacing in-between: $500 \mathrm{~nm}$ ) and open-channel (height: $400 \mathrm{~nm}$ ) glass chips, 
and reported that the diffusion coefficients of $50 \mathrm{~nm}$ diameter nanospheres in water were almost one-third of the theoretical value derived from the Stokes-Einstein equation. The authors consider this indirect proof of the higher viscosity of water in the channel compared to the bulk solution. In other work $[23,44]$ it was found that water in a $300 \mathrm{~nm}$-sized quartz glass nanochannel was a factor of three slower than predicted by the Hagen-Poiseuille law.

The effect of the small dimensions on viscosity also becomes apparent in capillary flow in nanochannels (i.e., flow driven by capillary forces). Tas et al. [45] found that for water in $50 \mathrm{~nm}$ high channels $(20 \mu \mathrm{m}$ wide), the apparent viscosity is $23 \pm 16 \%$ higher than the bulk viscosity, and $7 \pm 11 \%$ higher for $150 \mathrm{~nm}$ high $(20 \mu \mathrm{m}$ wide) channels. They argue that this is not due to increased ordering of the polar water molecules near the channel walls, as was proposed earlier by Churaev et al. [46], but is more likely caused by the electroviscous effect that arises from electro-osmotic counter flow induced by a streaming potential. The electroviscous effect is especially large under the conditions of double layer overlap; see, e.g., [26].

\section{Chemistry in nanoconfinement}

This review is mainly concerned with the changes in chemistry that are introduced by the new physics imposed by nanoconfinement. Most previous studies of chemistry in nanochannels and other nanostructures have focused on DNA. This topic has recently been reviewed by Eijkel et al. [25], and deals mainly with the separation of DNA fragments using entropic principles; for example in entropic traps [47], by confinement-induced [48] or hydrodynamic shear-induced entropic recoil [49], or by confinement in 2$\mathrm{D}$ nanochannels with diameters that are less than the persistence length of DNA (ca. $50 \mathrm{~nm}$ [50]). Another area of research related to this, and which also applies artificial nanostructures, is the study of the properties of single DNA molecules, including their base sequences [51].

It is obvious from this work on DNA in confinement that entropy is the main parameter involved in differences between the behavior of molecules in the bulk of the fluid and molecules confined in pores. Even if adsorption on the pore walls is not taken into account (this provides an extra contribution to the free energy of a fluid in a nanochannel compared to the bulk fluid), this behavior is what one would expect based on entropy arguments: molecules in a pore will lose some of the translational and rotational freedom they have in the bulk fluid, and the smaller the pore, the higher the loss of freedom. Logically, for a specific pore size, the entropic losses will be larger for larger (longer-chain) molecules such as synthetic polymers and biomolecules such as DNA and proteins.
Following this entropy-based reasoning, large effects of nanochannel confinement would be expected for those chemical processes in which large entropic changes are also involved in the bulk. Examples are chemical reactions that lead to a change in the number of molecules (like polymerization) and thus to a large change in the translational contribution to entropy, and possibly also in the rotational and vibrational parts. In general, one might expect reactions involving a large molar activation volume (i.e., the change in molar volume between reactants and the transition state) to demonstrate significant effects when carried out in confinement. These will typically be reactions that also exhibit a large pressure effect, like Diels-Alder cyclo-additions [52]. Large activation volume changes occur due to changes in the positions of the atoms during the formation of the transition state or due to specific solute-solvent interactions. The latter gives an additional activation volume change caused by electrostriction (i.e., the deformation of the solvent around the molecule or ion that is induced by the electric field of the polar molecule or ion), an example of which is the Baylis-Hillman reaction, which involves several steps in which ions are created or lost [52].

Large rate enhancements are observed for specific organic chemistry in water compared to organic solvents (e.g., for Michael reactions [53]), which are attributed to strong solvophobic interactions involving the hydrophobic packing of reactive molecules. Hydrophobic molecules are then located in cavities in the hydrogen-bonded network of water. The effect can even be larger than the effect of pressure.

Hardly any of the classes of chemical reactions mentioned above have been tested experimentally in confinement. A simple calculation of the feasibility of, say, observing any effect of nanoconfinement on reactions with a large activation volume leads to the conclusion that substantial effects can only be expected for nanochannels with diameters of below $1 \mathrm{~nm}$. This derives from the fact that activation volumes in the literature that can be considered large are in the range $30-60 \mathrm{~cm}^{3} \mathrm{~mol}^{-1}$, which yields a molecular volume of ca. $0.05-0.10 \mathrm{~nm}^{3}$ or a cubic molecular box with sides of $0.37-0.46 \mathrm{~nm}$. This is typical of the range of pore sizes in zeolites and some sol-gel silica glasses. It is thus no surprise that studies on reactions in zeolites are the only well-documented examples of chemistry in nanochannels. One of the most interesting topics studied in this area is shape selectivity (as mentioned earlier), which is simply the concept that the transformation of reactants depends on how these molecules fit to the active site of the catalyst, in this case the zeolite. Smit and Maesen have recently given a comprehensive overview of our current understanding of this phenomenon [54]. The basic mechanisms that give rise to shape selectivity are 
threefold: reactant shape selectivity, which consists of the idea that molecules that are too large to enter the pores do not reach active sites, and are therefore not converted into products; transition state shape selectivity, in which molecules and transition states that are too large to fit inside a pore will not form; and product shape selectivity, where new molecules are formed in the adsorbed phase, but are too large to desorb as a product. Figure 3 gives an example from the earlier work of Smit and coworkers, in which they introduce their basic ideas [55].

\section{Protein folding in confinement}

As was mentioned earlier, the largest effects of entropy on chemical reactions are expected for channels with diameters of $<1 \mathrm{~nm}$. One can also expect such effects for reactions in which conformational changes which reach the size of a nanochannel occur (i.e., for molecules in which one particular configuration has a size comparable to the nanochannel diameter). A typical example of such a reaction (or perhaps this is better termed a phase transition) is the folding of a protein. In their folded states, proteins can have sizes ranging from a few $\mathrm{nm}$ to ca. $8 \mathrm{~nm}$ (for BSA and hemoglobin; 67 and $64 \mathrm{kDa}$, respectively) up to sizes of ca. $15 \mathrm{~nm}$ for immunoglobulin $\mathrm{G}(\mathrm{IgG}, 150 \mathrm{kDa})$ or $65 \mathrm{~nm}$ for immunoglobulin M (IgM, $900 \mathrm{kDa})$. Their folding kinetics and paths of transformation are expected to be affected when they are introduced into a nanochannel which matches their size, and perhaps even when they are placed in much larger channels, at high loading. It is also known that enzymes need a certain flexibility in order to be able to perform their catalytic actions [56], and so biocatalysis with enzymes is also a good category of reactions to test in nanoconfinement.

One reason to study protein folding in nanochannel confinement is the following. In physiological conditions, a protein folds into a unique native structure. However, due to the crowded environment of the living cell, excluded volume effects play a significant role in the function, stability, and interactions of individual macromolecules. To create a crowded microenvironment, many researchers have started to encapsulate model proteins in silica glass using a sol-gel technique [57]. This sol-gel glass encapsulation is performed at room temperature, and the chemical conditions during processing do not denature most biomolecules, which makes this an attractive method of immobilizing the enzyme. The exact size of a protein-occupied pore in a solgel glass is not always known, but the average pore diameter in a wet-aged glass is usually a little over $10 \mathrm{~nm}$. In a study by Eggers et al. [58], the fraction of the total volume excluded by the silica matrix (ca. 14\%) is less than the fractional volume occupied by macromolecules in a living cell (estimated to be over 30\%).

In the study by Eggers et al. [58], the influence of solvent effects on the secondary structure of sol-gel silicaencapsulated proteins were examined. In many of the studied cases, the $\alpha$-helical content of the encapsulated protein was increased by adding solutes at concentrations that have no effect on the protein in the absence of the glass. The authors explain this by stating that perturbed water at the silica interface increases the average free
Fig. 3 Adsorption of linear and branched hexane isomers in AFI (left) and AET (right). The linear isomers are stretched in the smaller channel (AFI), while coiled conformations also occur in the wider channel (AET). The graphs show the projected endto-end distance distribution of $n$-hexane (red) and 2,2-DMB (black); the arrows indicate the effective size of the molecules. From [55]
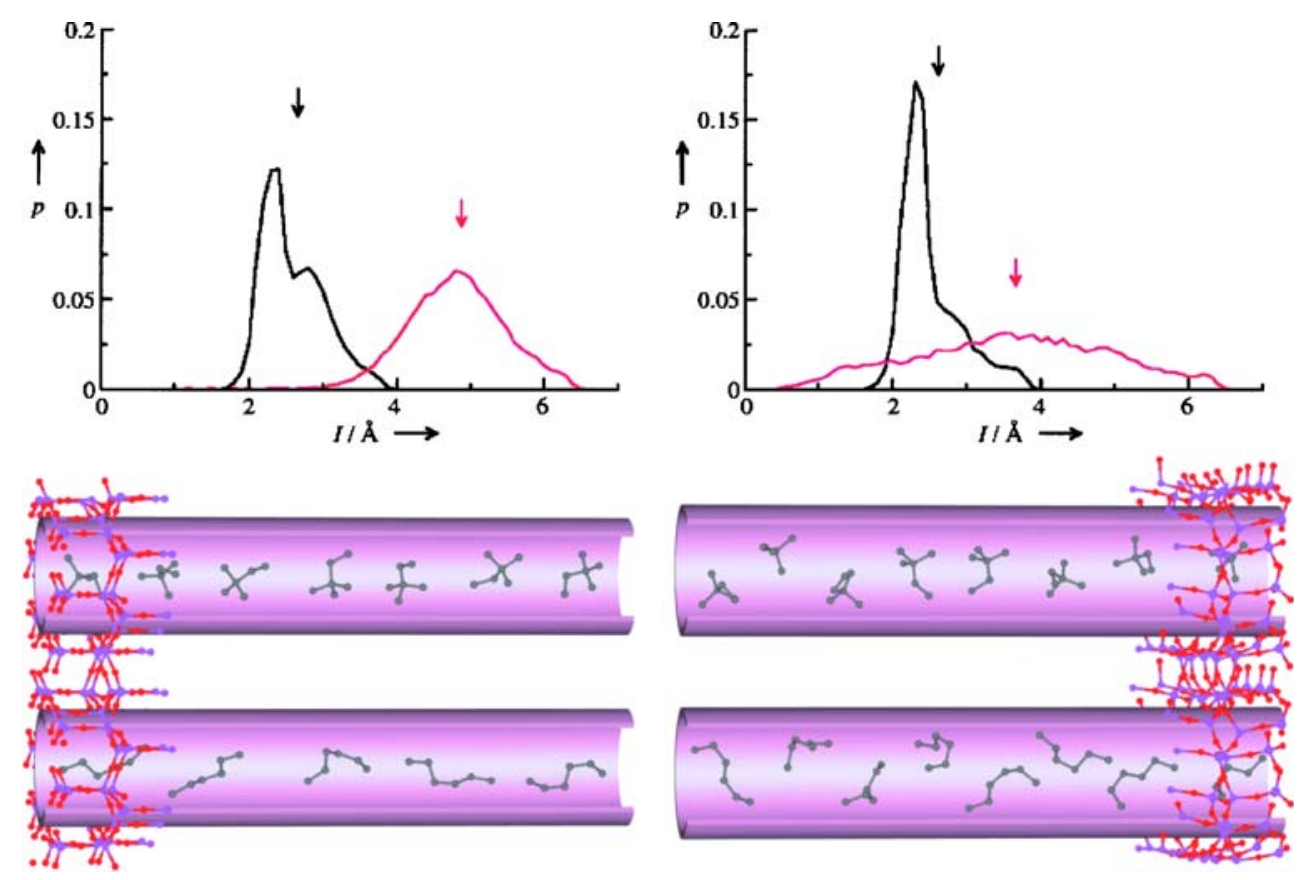
energy of the bulk water phase. The strength of the hydrophobic effect inside the glass matrix is therefore diminished, and the conformation of encapsulated proteins is destabilized. Solutes can increase the strength of the hydrophobic effect and influence folding equilibria without directly interacting with the protein.

Similar effects were observed by Menaa et al. [59]. However, these authors used modified siloxanes as host materials, and the modification was performed using monosubstituted alkoxysilanes with alkyl groups of increasing chain length. They found that apomyoglobin changes from an unfolded state to a native-like helical state as the content of the hydrophobic precursor increases from 0 to $15 \%$. At a fixed modifier content, the helical structure of apomyoglobin increased with the chain length of the $\mathrm{R}$ group, a trend also observed for the tertiary structure of ribonuclease A, which strongly suggests that protein folding is sensitive to the hydrophilic/hydrophobic balance of neighboring surfaces. The observed structural changes showed no correlation with the total surface area or average pore size (here between 3.8 and $8.9 \mathrm{~nm}$ with protein encapsulation) of the modified glasses.

According to Eggers [58], these results suggest that macromolecular crowding and molecular confinement are accompanied by hydration effects. These alter the stabilizing effect of the excluded volume on the protein structure, depending on the surface chemistry of the crowding agents and their influence on the bulk water structure.

Imai et al. [60] performed a theoretical study of the influence of hydration effects on the coil-to-native structural transition of protein $\mathrm{G}$ in aqueous solution, and found that a large gain in protein intramolecular energy is involved in folding, which is almost canceled out by a large loss in energy due to dehydration. The gain in hydration entropy is comparable with or even larger than the total energy gain. Coupled, these two factors are capable of suppressing the conformational entropy loss associated with folding, see Fig. 4. The conclusion is thus that the overall folding process is driven by water entropy changes.

In this respect, the work of Harano et al. [61] is also worth mentioning. They demonstrated that the folded state of the protein is favored because of a gain in the translational entropy (TE) of water. If the number of peptide residues in the protein is sufficiently large, the $\mathrm{TE}$ gain dominates over the conformational entropy loss upon folding. For protein G, they found that the largest TE is actually attained in the native structure.

A picture arises from the above considerations that confinement (as a mimic of the crowded environment) stabilizes the native folded state of a protein, at least for the proteins studied. In fact, it was found in simulation work that $\alpha$-hairpins and small proteins become stabilized by moderate confinement, such as confinement in spherical pores, but are destabilized in overconfinement (see [62] and references therein). The role of water in all this is crucial: the formation of a protein-water hydrogen bond decreases the translational entropy of a water molecule, and this entropy is directly related to the tube diameter $d$ (as was discussed in the sections on phase equilibria and transport in water-filled nanochannels). This is due to the fact that the hydrogen bond network becomes less extensive with decreasing pore size, and becomes negligible for extremely confined regions such as inside narrow carbon nanotubes. As the solvent entropy changes, the relative stability of peptide conformational states changes accordingly.

As we have done in every section, here we will also investigate the benefits of using micromachined nanochannels (e.g., with hydraulic diameters of between 10 and $100 \mathrm{~nm}$ ) for studies of protein folding or enzymatic chemistry. Little work has been done on this. Tsukahara et al. [63] have performed experiments with enzymes in microfluidic channels (100 $\mu \mathrm{m}$ wide, $40 \mu \mathrm{m}$ deep, and $40 \mathrm{~mm}$ long) and nanochannels (620 nm width, $234 \mathrm{~nm}$ depth, $200 \mu \mathrm{m}$ length), and compared the results with a reaction in bulk. The differences in reaction rates between

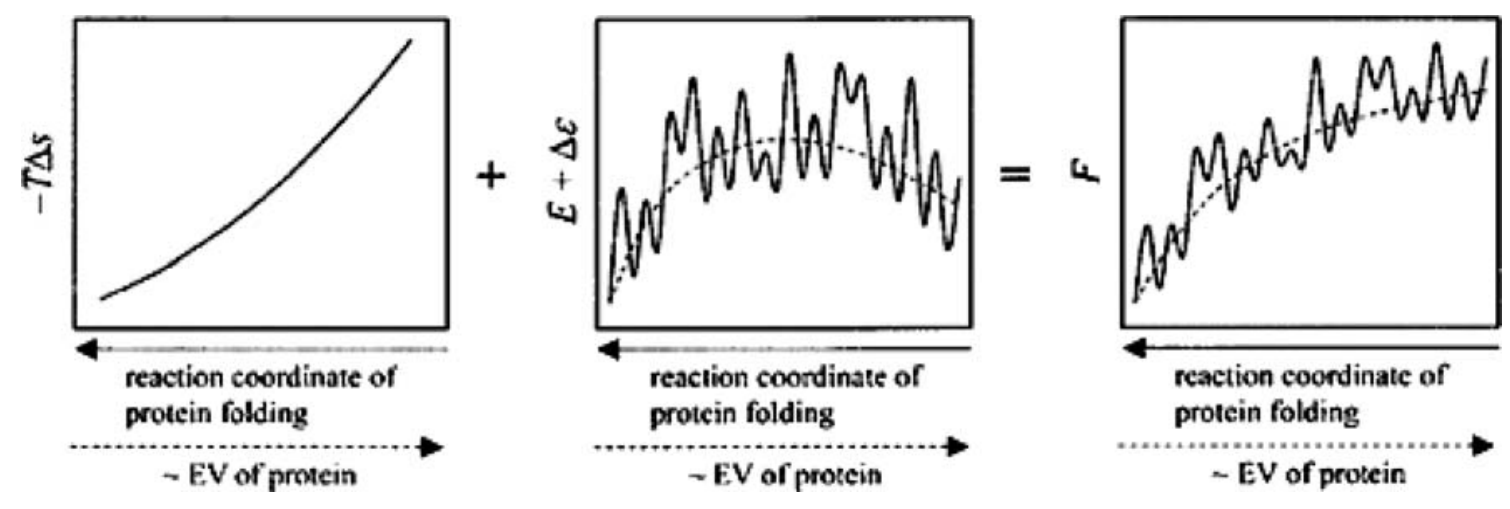

Fig. 4 Schematic illustration of free energy landscape of protein folding (right), which is a combination of entropic (left) and energetic (center) components along the folding reaction coordinate. Dashed line: global shape hidden in the ragged profile. From [60] 
the bulk and microfluidic channels were marginal, but the reaction rates and Michaelis constants were twice as high in the nanochannel as in the other systems. The authors present two explanations for the enhancement of the reaction rate in the nanochannel. The first is the adsorption of the enzymes onto the surface of the nanochannel; the second is related to the confinement of liquid. They discard the first explanation based on previous work, and state that the higher reaction rate must be due to the enhanced proton mobility in confined water, based on earlier NMR results (see the section on transport and [23]) which showed that the proton transfer rate was enhanced by a factor of more than ten compared to bulk water. Since the proton transfer rate will depend on the proton concentration, the effect of confinement was tested for the $\mathrm{pH}$ range 6.0-9.0. Maximum rates for all three cases occurred at $\mathrm{pH}$ 7.5 , but there was only a slight effect of $\mathrm{pH}$ on the rate in the nanochannel, so the activity enhancement remains unsolved.

Another interesting study is the work of Moorthy et al. [64], who used microchannels with hydrogel microstructures containing nanopores. The density of these nanopores can be changed by adjusting the cross-linking density in order to investigate protein-protein interactions in a nanoenvironment so as to mimic macromolecular crowding. Crowding or confinement by molecules or structures affects the equilibrium and kinetics of reactions, with a significant difference observed between the reactant-excluded volume and the product-excluded volume [65] (in fact, this is the "activation volume" discussed before). The microchannel in Moorthy's study can be used to adjust and control the microenvironment of the hydrogel and the proteins in it. It was found that the effect of crowding on the binding interaction depends very much on the intrinsic properties of the proteins.

\section{Nanochannel fabrication}

In a recent review, Perry and Kandlikar [66] discuss a number of fundamental methods of fabricating nanochannels based on standard semiconductor processing. Such methods include (i) bulk nanomachining and wafer bonding, where the nano dimension is defined by the etching depth in the bulk of, say, a glass or a silicon substrate (see, e.g., [67]), and (ii) surface nanomachining, where the nanodimension arises from a very thin sacrificial layer deposited on a solid substrate and covered with a (thicker) film (see, e.g., [68]). Both methods lead to nanochannels that have their smallest dimension perpendicular to the substrate (i.e., running parallel to the substrate surface); however, by combining them with deep-UV photolithography, laser interference lithography, or nanoimprint lithography (NIL)
$[69,70]$, it also becomes possible to control the lateral dimension (i.e., the dimension parallel to the substrate surface) down to several tens of nanometers. Alternative methods of achieving nano dimensions in the lateral direction without sophisticated lithography make use of shadowing effects at step edges [71, 72], the special structures that arise after stiction of a surface-machined cantilever [72], or of nonuniform film step coverage in deep trenches, followed by an annealing step that leads to reflow of the film material and shrinkage of the channel [73]. In these methods, the limitations on dimensional control derive from either the lithographic definition, the control over the thin film thickness (and its uniformity), the control over the etching depth (and its uniformity), or the surface roughness (which is usually below $0.5 \mathrm{~nm}$ on average when polished silicon or glass substrates are used). Nanochannels with dimensions down to ca. $10 \mathrm{~nm}$ in lateral size (i.e., parallel to the surface), and down to a few $\mathrm{nm}$ in vertical size (i.e., perpendicular to the substrate) can be achieved. Lithographically defined nanochannels that have lateral dimensions of $50 \mathrm{~nm}$, but depths of up to $40 \mu \mathrm{m}$ were recently reported by Mao et al. [74]; the fabrication process used was a combination of deep reactive ion etching and thin film growth to narrow the channels. The interfacing of these nanochannels with larger fluidic structures has been described in several of the papers that have already been mentioned in this paper, although we would like to add the excellent work by Cao et al., who managed to construct gradient nanostructures to connect microfluidics to nanochannels, to this list [75].

Besides these planar nanochannels (i.e., channels running parallel to the substrate surface), there are a number of ways to create nanochannels (and arrays thereof) that have channel directions perpendicular to the substrate surface. For example, photolithography can be used to define nanosized spaces between pillars [76], or silicon or aluminum can be treated electrochemically to achieve a porous layer with random but (if manufactured under the right conditions) vertically aligned pores, with typical pore sizes in the range $2-10 \mathrm{~nm}$ for porous silicon [77] and 30$70 \mathrm{~nm}$ for porous alumina [78]. Although it is not a trivial task to properly interface these channels to achieve fluid flow through them unless they are designed as membranes with open space on either side of each pore, these porous layers may also be useful for the studies described in this paper, and can actually be designed such that the processes that occur in the pores can be monitored with integrated analysis tools in situ (see below). The latter also holds for any method that can produce layers of an ordered porous material, like zeolites, mesoporous silica [79], or nanoporous metal-organic frameworks (MOFs) [80], in which also very interesting confinement effects have also been observed [81, 82]. 


\section{Analysis on nanochannels}

Because of the very small volume, and therefore the very small amounts of material in the nanochannels, it is not a trivial task to monitor the chemistry occurring in the nanochannels. The most sensitive method is fluorescence, although this is not a generally applicable technique because molecules must be labeled in order to be able to use it; this means that the very nature of the molecules to be studied may change such that they are no longer relevant for the chemical problem under study. Fluorescence has proven to be very useful for characterizing the properties of liquids [44], where it is mostly used as a tracer for velocity measurement or zone dispersion. It is essential in studies of DNA behavior and protein folding (in the latter, the fluorescence intensity depends on the folding state). The FRET (Förster resonance energy transfer [83]) conceptwhich describes an energy transfer mechanism between two chromophores where an excited donor chromophore can transfer energy by a nonradiative, long-range dipole-dipole coupling mechanism to an acceptor chromophore that is in close proximity, typically within the $10 \mathrm{~nm}$ range - can be used to study protein conformation in nanoconfinement.

Other optical techniques that are useful for spectroscopy are UV-Vis, IR and Raman. Because of the small dimensions of nanochannels, transmission measurements are not very useful, because the light path is simply too short to achieve reasonable signal-to-noise ratios. On the other hand, the concept of attenuated total internal reflection, which operates via the evanescent field on a planar waveguide, is very useful. An example of the use of multiple internal reflection IR in silica nanochannels of $100 \mathrm{~nm}$ width and 400-500 $\mathrm{nm}$ depth was recently published [84]. The authors used the IR to measure the adsorption of dyes to the nanochannel surface in a fieldeffect flow control device. The method would also be ideal for studying chemistry in nanochannels, in which case FTIR would be used to provide fingerprints of reactants and products.

The possibility of integrating electrical and optical elements is one of the main advantages of a "top-down" fabrication approach to nanochannels. State-of-the-art technology allows us to scale these elements to the dimensions of the nanochannels, and thus to scale them to the typical range of the signals one wishes to register from the nanosystem. Besides these sensor-like elements, many other components can of course be integrated too, such as mixers, heaters and concentration gradient-generating microfluidic networks, all of which are used to control the chemical and physical conditions of the liquid in the nanochannel.

Lithographically defined metal patterns - such as that consisting of a platinum thin film, as implemented in
[85] (or in [86]) - in nanochannels may serve several different purposes. They may act as electrodes for amperometry, conductometry, and electrolysis (e.g., to generate bubbles for mechanical actuation), or as driving electrodes for electro-osmotic flow, electrophoresis, or electrowetting.

A final example of an integrated system that will be useful for studying chemistry in nanochannels is a microfluidic stripline chip for NMR (nuclear magnetic resonance) [87]. In its current form, this device generates NMR spectra down to a concentration of ca. $1 \mathrm{mM}$ in a volume of $600 \mathrm{~nL}$ in ca. $12 \mathrm{~h}$ using a $600 \mathrm{MHz}$ machine. This concentration falls within the range of what is typically used in NMR studies on proteins, but for nanoconfinement studies the signal-to-noise ratio needs to be improved so that the concentration limit of detection will fall within the $\mu \mathrm{M}$ range, which is what our group is currently working on. The chip currently has a relatively large microchannel in it, but it is technologically feasible to replace this channel with an array of nanochannels, which allows the same "filling factor" to be retained for the NMR stripline and does not compromise the NMR measurement quality. When applying NMR to protein folding in such systems, spin relaxation measurements would be the best option (see, e.g., [88]). The use of a microfluidic network attached to the nanochannel array would allow protein behavior to be studied as a function of parameters such as $\mathrm{pH}$, specific concentration variations, pressure, temperature, shear rate, etc.

\section{Conclusions}

In this review we have investigated what is known about the chemistry (in particular, synthetic chemistry and protein folding processes) of nanochannels of different sizes. Among the different classes of pores-micropores (diameter below $2 \mathrm{~nm}$ ), mesopores (2-50 nm) and macropores (above $50 \mathrm{~nm}$ ) - most work has been devoted to micropores. Topics that have been studied in particular depth for this class of pores are phase transitions, water behavior and shape selectivity in the field of (petro)chemistry. The smaller mesopores, $2-10 \mathrm{~nm}$, are of particular interest for studies on protein folding in confinement (i.e., mimicking folding behavior in crowded solutions). Phenomena like single-file diffusion and charge exclusion-which are observed for water in micropores-do not occur for macropores (which cover the range that is accessible to modern microfabrication technology), but a few interesting findings have been reported for macropores, such as higher viscosity and very specific proton transport. A very brief overview of lithography-based fabrication methods for nanochannels was given, and finally the use of a number 
of (mainly spectroscopic) measurement methods was described, of which NMR striplines, optical and IR measurements via evanescent fields, and electrochemistry via nanoelectrodes are seen as very promising methods for future work on proteins and other molecules in nanoconfinement.

Open Access This article is distributed under the terms of the Creative Commons Attribution Noncommercial License which permits any noncommercial use, distribution, and reproduction in any medium, provided the original author(s) and source are credited.

\section{References}

1. McNaught AD, Wilkinson A (eds)(1997) IUPAC compendium of chemical terminology, 2nd edn. IUPAC, Research Triangle Park

2. Gelb LD, Gubbins KE, Radhakrishnan R, Sliwinska-Bartkowiak M (1999) Phase separation in confined systems. Rep Prog Phys 62:1573-1659

3. Broers AN (1988) Resolution limits for electron-beam lithography. IBM J Res Dev 32:502-513

4. Warnock J, Awschalom DD, Schafer MW (1986) Geometrical supercooling of liquids in porous glass. Phys Rev Lett 57:17531756

5. Tell JL, Maris HJ (1983) Specific heats of hydrogen, deuterium, and neon in porous Vycor glass. Phys Rev B 28:5122-5125

6. Fisher LR, Gamble RA, Middlehurst J (1981) The Kelvin equation and the capillary condensation of water. Nature 290:575-576

7. Fisher LR, Israelachvili JN (1979) Direct experimental verification of the Kelvin equation for capillary condensation. Nature 277:548-549

8. Evans R (1990) Fluids adsorbed in narrow pores: phase equilibria and structure. J Phys Cond Matter 2:8989-9007

9. Morishige K, Shikimi M (1998) J Chem Phys 108:7821-7824

10. Zarragoicoechea GJ, Kuz VA (2004) Critical shift of a confined fluid in a nanopore. Fluid Phase Equil 220:7-9

11. De Simone JM (2002) Practical approaches to green solvents. Science 297:799-802

12. Morishige K, Fujii H, Uga M, Kinukawa D (1997) Capillary critical point of argon, nitrogen, oxygen, ethylene, and carbon dioxide in MCM-41. Langmuir 13:3494-3498

13. Rothschild LJ, Mancinelli RL (2001) Life in extreme environments. Nature 409:1092-1101

14. Gallo P, Ricci MA, Rovere M (2002) Layer analysis of the structure of water confined in vycor glass. J Chem Phys 116:342346

15. Holt JK (2008) Methods for probing water at the nanoscale. Microfluid Nanofluid 5:425-442

16. Murata K, Mitsuoka K, Hirai T, Walz T, Agre P, Heymann JB, Engel A, Fujiyoshi Y (2000) Structural determinants of water permeation through aquaporin-1. Nature 407:599-605

17. Kolesnikov AI, Zanotti JM, Loong CK, Thiyagarajan P, Moravsky AP, Loutfy RO, Burnham CJ (2004) Anomalously soft dynamics of water in a nanotube: a revelation of nanoscale confinement. Phys Rev Lett 93:035503

18. Hummer G, Rasaiah JC, Noworyta JP (2001) Water conduction through the hydrophobic channel of a carbon nanotube. Nature 414:188-190
19. Fang H, Wan R, Gong X, Lu H, Li S (2008) Dynamics of single-file water chains inside nanoscale channels: physics, biological significance and applications. J Phys D Appl Phys 41:103002

20. Farrer RA, Fourkas JT (2003) Liquids confined in nanoporous sol-gel glasses. Acc Chem Res 36:605-612

21. Takahara S, Kittaka S, Mori T, Kuroda Y, Takamuku T, Yamaguchi T (2008) Neutron scattering and dielectric studies on dynamics of methanol and ethanol confined in MCM-41. J Phys Chem C 112:14385-14393

22. Kittaka S, Iwashita T, Serizawa A, Kranishi M, Takahara S, Kuroda Y, Mori T, Yamaguchi T (2005) Low temperature properties of acetonitrile confined in MCM-41. J Phys Chem B 109:23162-23169

23. Tsukahara T, Hibara A, Ikeda Y, Kitamori T (2007) NMR study of water molecules confined in extended nanospaces. Angew Chem Int Ed 46:1180-1183

24. Tiggelaar RM, Benito-López F, Hermes DC, Rathgen H, Egberink RJM, Mugele FG, Reinhoudt DN, van den Berg A, Verboom W, Gardeniers HJGE (2007) Fabrication, mechanical testing and application of high-pressure glass microreactor chips. Chem Eng J 131:163-170

25. Eijkel JCT, van den Berg A (2005) Nanofluidics: what is it and what can we expect from it? Microfluid Nanofluid 1:249267

26. Schoch RB, Han J, Renaud P (2008) Transport phenomena in nanofluidics. Rev Mod Phys 80:839-883

27. Abgrall P, Nguyen NT (2008) Nanofluidic devices and their applications. Anal Chem 80:2326-2341

28. Baldessari F (2008) Electrokinetics in nanochannels. Part I. Electric double layer overlap and channel-to-well equilibrium. J Colloid Interf Sci 325:526-538

29. Strathmann H (2004) Ion-exchange membrane separation processes (Membrane Science and Technology, vol 9). Elsevier, Amsterdam

30. Doyle DA, Morais Cabral J, Pfuetzner RA, Kuo A, Gulbis JM, Cohen SL, Chait BT, MacKinnon R (1998) The structure of the potassium channel: molecular basis of $\mathrm{K}+$ conduction and selectivity. Science 280:69-77

31. Wang X, Kang J, Wang S, Lu JJ, Liu S (2008) Chromatographic separations in a nanocapillary under pressure-driven conditions. $J$ Chromatogr A 1200:108-113

32. Liu S, Pu Q, Gao L, Korzeniewski C, Matzke C (2005) From nanochannel-induced proton conduction enhancement to a nanochannel-based fuel cell. Nano Lett 5:1389-1393

33. Schoch RB, Cheow LF, Han J (2007) Electrical detection of fast reaction kinetics in nanochannels with an induced flow. Nano Lett 7:3895-3900

34. Levitt DG (1973) Dynamics of a single-file pore: non-Fickian behavior. Phys Rev A 8:3050-3054

35. Kukla V, Kornatowski J, Demuth D, Girnus I, Pfeifer H, Rees LVC, Schunk S, Unger KK, Kärger J (1996) NMR studies of single-file diffusion in unidimensional channel zeolites. Science 272:702-704

36. Smit B, Maesen TLM (2008) Molecular simulations of zeolites: adsorption, diffusion, and shape selectivity. Chem Rev 108:41254184

37. Krishna R, Wesselingh JA (1997) The Maxwell-Stefan approach to mass transfer. Chem Eng Sci 52:861-911

38. Kievsky YY, Carey B, Naik S, Mangan N, ben-Avraham D, Sokolov I (2008) Dynamics of molecular diffusion of rhodamine $6 \mathrm{G}$ in silica nanochannels. J Chem Phys 128: 151102

39. Majumder M, Chopra N, Andrews R, Hinds BJ (2005) Nanoscale hydrodynamics: Enhanced flow in carbon nanotubes. Nature 438:44; erratum 930 
40. Yoo K, Tsekov R, Smirniotis PG (2003) Experimental proof for resonant diffusion of normal alkanes in LTL and ZSM-12 zeolites. J Phys Chem B 107:13593-13596

41. Chung S-H, Allen TW, Hoyles M, Kuyucak S (1999) Permeation of ions across the potassium channel: Brownian dynamics studies. Biophys J 77:2517-2533

42. Martin F, Walczak R, Boiarski A, Cohen M, West T, Cosentino C, Shapiro J, Ferrari M (2005) Tailoring width of microfabricated nanochannels to solute size can be used to control diffusion kinetics. J Control Release 102:123-133; corrigendum 183

43. Kaji N, Ogawa R, Oki A, Horiike Y, Tokeshi M, Baba Y (2006) Study of water properties in nanospace. Anal Bioanal Chem 386:759-764

44. Hibara A, Saito T, Kim H-B, Tokeshi M, Ooi T, Nakao M, Kitamori T (2002) Nanochannels on a fused-silica microchip and liquid properties investigation by time-resolved fluorescence measurements. Anal Chem 74:6170-6176

45. Tas NR, Haneveld J, Jansen HV, Elwenspoek M, van den Berg A (2004) Capillary filling speed of water in nanochannels. Appl Phys Lett 85:3274-3276

46. Churaev NV, Sobolev VD, Zorin ZM (1971) Special discussion on thin liquid films and boundary layers (held at University of Cambridge, 1970). Academic Press for the Faraday Society, London, pp 213-220

47. Han J, Turner SW, Craighead HG (1999) Entropic trapping and escape of long DNA molecules at submicron size constriction. Phys Rev Lett 83:1688-1691

48. Turner SWP, Cabodi M, Craighead HG (2002) Confinementinduced entropic recoil of single DNA molecules in a nanofluidic structure. Phys Rev Lett 88:128103

49. Wong PK, Lee Y-K, Ho C-M (2003) Deformation of DNA molecules by hydrodynamic focusing. J Fluid Mech 497:55-65

50. Tegenfeldt JO, Cao H, Reisner WW, Prinz C, Austin RH, Chou SY, Cox EC, Sturm JC (2004) Stretching DNA in nanochannels. Biophys J 86:596A

51. Tegenfeldt JO, Prinz C, Cao H, Huang RL, Austin RH, Chou SY, Cox EC, Sturm JC (2004) Micro- and nanofluidics for DNA analysis. Anal Bioanal Chem 378:1678-1692

52. Jenner G (2002) Comparative activation modes in organic synthesis. The specific role of high pressure. Tetrahedron 58:5185-5202

53. Jenner G (1995) Kinetic effects in water and ethylene glycol. Application to high pressure organic synthesis. New J Chem 19:173-178

54. Smit B, Maesen TLM (2008) Towards a molecular understanding of shape selectivity. Nature 451:671-678

55. Schenk M, Calero S, Maesen TLM, van Benthem LL, Verbeek MG, Smit B (2002) Understanding zeolite catalysis: inverse shape selectivity revised. Angew Chem Int Ed 41:2499-2502

56. Benkovic SJ (2003) A perspective on enzyme catalysis. Science 301:1196-1202

57. Avnir D, Braun S, Lev O, Ottolenghi M (1994) Enzymes and other proteins entrapped in sol-gel materials. Chem Mater 6:16051614

58. Eggers DK, Valentine JS (2001) Crowding and hydration effects on protein conformation: a study with sol-gel encapsulated proteins. J Mol Biol 314:911-922

59. Menaa B, Herrero M, Rives V, Lavrenko M, Eggers DK (2008) Favourable influence of hydrophobic surfaces on protein structure in porous organically-modified silica glasses. Biomaterials 29:2710-2718

60. Imai T, Harano Y, Kinoshita M, Kovalenko A, Hirata F (2007) Theoretical analysis on changes in thermodynamic quantities upon protein folding: essential role of hydration. J Chem Phys $126: 225102$
61. Harano Y, Kinoshita M (2005) Translational-entropy gain of solvent upon protein folding. Biophys J 89:2701-2710

62. Sorin EJ, Pande VS (2006) Nanotube confinement denatures protein helices. J Am Chem Soc 128:6316-6317

63. Tsukahara T, Mawatari K, Hibara A, Kitamori T (2008) Development of a pressure-driven nanofluidic control system and its application to an enzymatic reaction. Anal Bioanal Chem 391:2745-2752

64. Moorthy J, Burgess R, Yethiraj A, Beebe D (2007) Microfluidic based platform for characterization of protein interactions in hydrogel nanoenvironments. Anal Chem 79:5322-5327

65. Minton AP (2001) The influence of macromolecular crowding and macromolecular confinement on biochemical reactions in physiological media. J Biol Chem 276:10577-10580

66. Perry JL, Kandlikar SG (2006) Review of fabrication of nanochannels for single phase liquid flow. Microfluid Nanofluid 2:185-193

67. Haneveld J, Jansen H, Berenschot E, Tas N, Elwenspoek M (2003) Wet anisotropic etching for fluidic 1D nanochannels. J Micromech Microeng 13:S62-S66

68. Stern MB, Geis MW, Curtin JE (1997) Nanochannel fabrication for chemical sensors. J Vac Sci Technol B 15:2887-2891

69. Guo LJ (2004) Topical review: recent progress in nanoimprint technology and its applications. J Phys D Appl Phys 37:R123R141

70. Guo LJ, Cheng X, Chou C (2004) Fabrication of size-controllable nanofluidics channels by nanoimprinting and its applications for DNA stretching. Nano Lett 4:69-73

71. Kim DH, Sung S-K, Sim JS, Kim KR, Lee JD, Park B-G, Choi BH, Hwang SW, Ahn D (2001) Single-electron transistor based on a silicon-on-insulator quantum wire fabricated by a side-wall patterning method. Appl Phys Lett 79:3812-3814

72. Tas NR, Berenschot JW, Mela P, Jansen HV, Elwenspoek M, van den Berg A (2002) 2D-confined nanochannels fabricated by conventional micromachining. Nano Lett 2:1031-1032

73. Callender CL, Ledderhof CJ, Dumais P, Blanchetière C, Noad JP (2005) Fabrication of microchannel arrays in borophosphosilicate glass. J Mater Res 20:759-764

74. Mao P, Han J (2009) Massively-parallel ultra-high-aspect-ratio nanochannels as mesoporous membranes. Lab Chip 9:586-591

75. Cao H, Tegenfeldt JO, Austin RH, Chou SY (2002) Gradient nanostructures for interfacing microfluidics and nanofluidics. Appl Phys Lett 81:3058-3060

76. Baba M, Sano T, Iguchi N, Iida K, Sakamoto T, Kawaura H (2003) DNA size separation using artificially nanostructured matrix. Appl Phys Lett 83:1468-1470

77. Bomchil G, Herino R, Barla K, Pfister JC (1983) Pore size distribution in porous silicon studied by adsorption isotherms. $\mathrm{J}$ Electrochem Soc 130:1611-1614

78. Cai A, Zhang H, Hua H, Zhang Z (2002) Direct formation of selfassembled nanoporous aluminium oxide on $\mathrm{SiO}_{2}$ and $\mathrm{Si}$ substrates. Nanotechnology 13:627-630

79. Beck JS, Vartuli JC, Roth WJ, Leonowicz ME, Kresge CT, Schmitt KD, Chu CTW, Olson DH, Sheppard EW (1992) A new family of mesoporous molecular sieves prepared with liquid crystal templates. J Am Chem Soc 114:10834-10843

80. Li H, Eddaoudi M, O’Keefe M, Yaghi OM (1999) Design and synthesis of an exceptionally stable and highly porous metalorganic framework. Nature 402:276-279

81. Pan L, Olson DH, Ciemnolonski LR, Heddy R, Li J (2006) Separation of hydrocarbons with a microporous metal-organic framework. Angew Chem Int Ed 45:616-619

82. Finsy V, Verelst H, Alaerts L, De Vos D, Jacobs PA, Baron GV, Denayer JFM (2008) Pore filling dependent selectivity effects in the vapor phase separation of xylene isomers on the metal-organic framework MIL-47. J Am Chem Soc 130:7110-7118 
83. Förster T (1948) Zwischenmolekulare Energiewanderung und Fluoreszenz. Ann Physik 437:55-75

84. Oh Y-J, Gamble TC, Leonhardt D, Chung C-H, Brueck SRJ, Ivory CF, Lopez GP, Petse DN, Han SM (2008) Monitoring FET flow control and wall adsorption of charged fluorescent dye molecules in nanochannels integrated into a multiple internal reflection infrared waveguide. Lab Chip 8:251-258

85. Sparreboom W, Eijkel JCT, Bomer J, van den Berg A (2008) Rapid sacrificial layer etching for the fabrication of nanochannels with integrated metal electrodes. Lab Chip 8:402-407
86. Nichols KP, Eijkel JCT, Gardeniers H (2008) Nanochannels in SU-8 with floor and ceiling metal electrodes and integrated microchannels. Lab Chip 8:173-175

87. van Bentum PJM, Jansen JWG, Kentgens APM, Bart J, Gardeniers JGE (2007) Stripline probes for NMR. J Magn Res 189:104113

88. Lundström P, Mulder FAA, Akke M (2005) Correlated dynamics of consecutive residues reveal transient and cooperative unfolding of secondary structure in proteins. Proc Natl Acad Sci USA 102:16984-16989 